\title{
PSEUDOCOMPACT ALGEBRAS, PROFINITE GROUPS AND CLASS FORMATIONS
}

\author{
BY ARMAND BRUMER ${ }^{1}$
}

Communicated by A. Rosenberg, October 7, 1965

This note announces the main results obtained in a paper of the same title to appear in the Journal of Algebra, in which complete proofs can be found.

Introduction. We recall that a topological group $G$ is a profinite group if it is the inverse limit of finite groups and that a $G$-module $A$ is a discrete $G$-module if $A=\mathrm{U} A_{H}$, where $H$ runs through the open subgroups of $G$ and $A_{H}$ is the set of elements of $A$ left fixed by $H$ (cf. [4]). We note that if $H$ is a normal subgroup of $K$, then $A_{H}$ is a $K / H$-module. A class formation consists of a profinite group $G$ and a $G$-module satisfying certain axioms which we do not repeat here: the reader will find them and their consequences in [1]. The reciprocity map for the formation gives a homomorphism

$$
\omega_{H}: A_{H} \rightarrow H / H^{\prime}
$$

for each open subgroup $H$ of $G$ since $H / H^{\prime}$ is the group of the maximal abelian extension of $H$ (cf. p. 179 of [6]). Let $C_{H}$ be the kernel of $\omega_{H}$ and let $D_{H}$ be its cokernel. For each subgroup $K$ of $G$, containing $H$ as a normal subgroup, the exact sequence of $K / H$-modules

$$
0 \rightarrow C_{H} \rightarrow A_{H} \rightarrow H / H^{\prime} \rightarrow D_{H} \rightarrow 0
$$

gives rise to homomorphisms

$$
d_{q}: \hat{H}^{q-2}\left(K / H, D_{H}\right) \rightarrow \hat{H}^{q}\left(K / H, C_{H}\right)
$$

as the composition of two coboundary maps.

THEOREM 1. The following are equivalent for a class formation:

(i) $\operatorname{scd}_{p} G \leqq 2$,

(ii) For every integer $q$, the map $d_{q}$ induces an isomorphism onto on the p-primary components.

The second condition is equivalent to a group theoretic property introduced by Kawada in [3].

For any field $k$, let $G_{k}$ denote the Galois group of the separable closure of $k$. The following results about the associated class forma-

1 This paper was written with partial support of NSF grant GP4124 while the author was T. H. Hildebrandt Instructor at the University of Michigan. 
tions are proved in class field theory (cf. [1]).

(a) For a local field $k$, the reciprocity map is injective and its cokernel is $\hat{\boldsymbol{Z}} / \boldsymbol{Z}$, where $\hat{\boldsymbol{Z}}$ is the total completion of the integers. In particular, the cokernel is uniquely divisible and thus cohomologically trivial.

(b) For a function field of one variable over a finite field, the situation is as in (a).

(c) For a number field $k$, the reciprocity map is surjective and its kernel is the connected component of the identity of the idèle class group. The cohomology groups of the kernel are thus elementary 2groups and trivial if $k$ is totally imaginary.

(d) If $k$ is a field complete under a discrete rank one valuation with algebraically closed residue class field, the reciprocity map is an isomorphism [5].

It is not difficult to verify that cd $G_{k}=2$ in these examples, e.g. page II-16 of [4]. Tate has shown the stronger result scd $G_{k}=2$ for (a), (b) and (c) as a consequence of a deep duality: a proof is given on page II-25 of [4] for p-adic fields while the other cases remain unpublished.

Whenever a new class formation is discovered, a description of the kernel and cokernel of the reciprocity map is given and Theorem 1 tells us immediately whether or not $\operatorname{scd}_{p} G_{k}=2$ : this is the case in the above examples, except if $p=2$ and $k$ is a number field which is not totally imaginary.

Pseudocompact algebras. A complete Hausdorff topological ring $\Lambda$ is said to be a pseudocompact ring if it admits a system of open neighborhoods of 0 consisting of two sided ideals $I$ for which $\Lambda / I$ is an Artin ring. If $\Lambda$ is an algebra over the commutative pseudocompact ring $\Omega$, we shall say that $\Lambda$ is a pseudocompact $\Omega$-algebra if $\Lambda / I$ has finite length as $\Omega$-module. A complete Hausdorff topological $\Lambda$-module $M$ is pseudocompact if it admits a base of open neighborhoods of 0 consisting of submodules $N$ for which $M / N$ has finite length. The category $\mathfrak{C}_{\boldsymbol{\Lambda}}$ of pseudocompact $\Lambda$-modules is an abelian category with exact inverse limits and enough projectives: in fact, we show that $\mathcal{C}_{\Lambda}$ is dual to the category $D_{\Lambda^{0}}$ of discrete modules over the opposed algebra $\Lambda^{0}$. We may thus define as usual the homological dimension, $\operatorname{hd}_{\Lambda}(A)$, of the pseudocompact $\Lambda$-module $A$ as the least integer $n$ for which we can find a projective resolution $0 \rightarrow P_{n} \rightarrow \cdots \rightarrow P_{0} \rightarrow A \rightarrow 0$ and we write $\operatorname{gldim} \Lambda=\sup _{A} \operatorname{hd}_{\Lambda}(A)$ where $A$ runs through $\mathfrak{C}_{\Lambda}$. It is possible to introduce functors $\operatorname{Ext}_{\Lambda}^{n}: \mathfrak{C}_{\Lambda} \times \mathscr{D}_{\Lambda} \rightarrow D_{\Omega}$ and $\operatorname{Tor}_{n}^{\Lambda}: \mathfrak{C}_{\Lambda^{0}} \times \mathcal{C}_{\Lambda}$ 
$\rightarrow \mathfrak{C}_{\Omega}$ as derived functors of $\mathrm{Hom}_{\Lambda}$ and of the completed tensor product. These allow us to generalize the elementary results on homological dimension in complete noetherian semilocal rings. As a first application we generalize a result proved by P. M. Cohn in case $\Omega$ is a field [2].

THEOREM 2. Let $\Omega$ be a commutative pseudocompact ring and let $\Omega\left\{\left\{x_{i}\right\}\right.$ be the algebra of noncommuting formal power series in $\left\{x_{i}\right\}$ over $\Omega$. Then

$$
\operatorname{gldim} \Omega\left\{\left\{x_{i}\right\}\right\}=\operatorname{gldim} \Omega+1 .
$$

Let $G$ be a profinite group and let $\Omega$ be a commutative pseudocompact ring. The complete group algebra $\Omega[[G]]$ of $G$ over $\Omega$ is defined by $\Omega[[G]]=$ proj $\lim \Omega[G / U]$ where $U$ runs through the open normal subgroups of $G$ and $\Omega[G / U]$ is the ordinary group algebra. By construction, $\Omega[[G]]$ is a pseudocompact $\Omega$-algebra. For any discrete $\Omega[[G]]$-module $A, \operatorname{Ext}_{\Omega[[G]]}^{n}(\Omega, A)=H^{n}(G, A)$ while $\operatorname{Tor}_{n}^{\Omega[[G]]}(B, \Omega)$ define homology groups for $G$ with coefficients in the pseudocompact $\Omega[[G]]$-module $B$.

TheOREM 3. Let $\mathrm{cd}_{\Omega} G$ denote the $\sup$ of $\operatorname{cd}_{p} G$ over the primes $p$ which are not units in $\Omega$. Then

$$
\operatorname{gldim} \Omega[[G]]=\operatorname{gldim} \Omega+\operatorname{cd}_{\Omega} G .
$$

Application to profinite groups and Theorem 1 . We prove Theorem 1 by presenting $G$ as the quotient $F / N$ of a free profinite group modulo a closed normal subgroup $N$. The following criterion, proved by the ideas of the last section, plays a crucial role and may be of independent interest.

THEOREM 4. Let $F$ be a free profinite group. The following are equivalent for $G=F / N$.

(i) $\operatorname{scd}_{p} G \leqq 2$.

(ii) $N / N^{\prime}$ is a projective $\hat{\boldsymbol{Z}}_{p}[[G]]$-module and the $p$-Sylow subgroup of the abelian profinite group $N \cap V^{\prime} /[N, V]$ is trivial for every open subgroup $V$ of $F$ containing $N$. Here $[N, V]$ is the closed subgroup generated by the commutators $[n, v], n \in N, v \in V$ and $\hat{\boldsymbol{Z}}_{p}$ is the ring of p-adic integers.

\section{BIBLIOGRAPHY}

1. E. Artin and J. Tate, Class field theory, Mimeographed notes, Harvard University, Cambridge, Mass., 1961. 
2. P. M. Cohn, Unique factorization in noncommutative power series rings, Proc. Cambridge Philos. Soc 58 (1962), 452-464.

3. Y. Kawada, Cohomology of group extensions, J. Fac. Sci. Univ. Tokyo Sect. I 9 (1963), 417-431.

4. J.-P. Serre, Cohomologie Galoisienne, Lecture Notes in Mathematics No. 5, Springer, Berlin, 1964.

5. —- Corps locaux et isogénies, Séminaire Bourbaki, 1958/1959, Éxposé 185

6. - Corps locaux, Hermann, Paris, 1962.

UNIVERSITY OF MichigAN

\section{COHOMOLOGY OF DIHEDRAL GROUPS OF ORDER $2 p$}

\section{BY PHYLLIS GRAHAM}

Communicated by G. Whaples, November 29, 1965

Let $D$ be a dihedral group of order $2 p$, where $p$ is an odd prime. $D$ is generated by the elements $\alpha$ and $\beta$ with the relations $\alpha^{p}=\beta^{2}=1$ and $\beta \alpha \beta=\alpha^{-1}$. Let $A$ be the subgroup of $D$ generated by $\alpha$, and let $A_{0}, A_{1}, \cdots, A_{p-1}$ be the subgroups generated by $\beta, \alpha \beta, \cdots, \alpha^{p-1} \beta$, respectively. Let $M$ be any $D$-module. Then the cohomology groups $H^{n}\left(A_{0}, M\right)$ and $H^{n}\left(A_{i}, M\right), i=1,2, \cdots, p-1$ are isomorphic for every integer $n$, so the eight groups $H^{-1}(D, M), H^{0}(D, M), H^{1}(D, M)$, $H^{2}(D, M), H^{-1}(A, M), H^{0}(A, M), H^{-1}\left(A_{0}, M\right)$, and $H^{0}\left(A_{0}, M\right)$ determine all cohomology groups of $M$ with respect to $D$ and to all of its subgroups. We have found what values this array takes on as $M$ runs through all finitely generated $D$-modules.

All possibilities for the first four members of this array are determined as a special case of the results of Yang [4]. But we have not been able to extend his methods so as to determine all possibilities for the whole array; our methods are independent to those of Yang.

Method of Proof. First we follow the method of Parr [3] in showing that it suffices to consider only finitely generated $\tilde{Z}$-torsion free $\tilde{Z} D$-modules, where $\tilde{Z}$ is the ring of all fractions $m / n$ of rational integers $m$ and $n$ such that $(n, 2 p)=1$. Lee [2] has listed all indecomposable modules of this type; there are ten. We compute directly several of the cohomology groups for the first five modules in her list. The last five of her list may be treated as members of extensions. These yield exact sequences of cohomology groups, which give information about the last five modules. We can then complete the values in all of the arrays by using the result that the cohomology of 